\title{
PHYSICAL VARIATIONAL PRINCIPLES WHICH SATISFY THE PALAIS-SMALE CONDITION
}

\author{
BY WILLIAM B. GORDON ${ }^{1}$ \\ Communicated by Philip Hartman, February 9, 1972
}

1. Using variational techniques we have found conditions which insure the existence of trajectories to conservative dynamical systems which wind around singularities of the potential and are of the following four types: (1a) periodic trajectories cutting arbitrary small neighborhoods of the singularities; (1b) periodic trajectories having arbitrary given period; (2a) trajectories joining two fixed points and cutting arbitrarily small neighborhoods of the singularities; $(2 \mathrm{~b})$ trajectories joining two fixed points with arbitrary given time of transit.

The trajectories are obtained as paths at which certain functionals attain extreme values. These functionals satisfy the Palais-Smale condition, and the trajectories are computable by the Ritz method. At present we must impose a certain condition on the potential which excludes the gravitational case, and the P-S condition is definitely not satisfied by these functionals in this case. Moreover, our theorems provide for the existence of trajectories of a type which cannot occur in the gravitational case.

On the other hand, our theorems apply to planar $n$-body systems consisting of particles which attract each other with forces that are (roughly) inverse cube or stronger in a neighborhood of each particle. Hence, e.g., we obtain periodic solutions to such systems which are very complicated but have arbitrarily long or short periods.

2. Consider the dynamical equation

$$
\ddot{x}+\nabla V(x)=0,
$$

where $x=\left(x^{1}, \ldots, x^{N}\right)$ denotes a general point of $R^{N}$ and $V=V(x)$ is a real valued function on $R^{N}$ with gradient $\nabla V$. It is assumed that $V$ is of class $C^{2}$ everywhere on $R^{N}$ except at a nonempty closed set of points $S$ at which $V$ has infinitely deep wells; i.e., we suppose that $V(x) \rightarrow-\infty$ as $x \rightarrow S$. We shall at times assume one or more of the following conditions. (The first condition is always assumed, and this is the condition which excludes the gravitational case. See $\S 4$.)

AMS 1970 subject classifications. Primary 70H25, 58F05; Secondary 58E99.

${ }^{1}$ Mathematics Research Center, Naval Research Laboratory, Code 7840, Washington, D. C. 20390 
[A] There exists a $C^{2}$ function $U$ which is defined on some open neighborhood $N$ of $S$, has infinitely deep wells at $S$, and satisfies

$$
-V(x) \geqq|\nabla U(x)|^{2} \quad \text { for all } x \text { in } N-S \text {. }
$$

[B] For each $p$ in $\partial S,(x-p) \cdot \nabla V(x)>0$ for all $x$ sufficiently close to $p$ and not in $S$.

[C] $V$ is bounded above; say, $V \leqq 0$.

If these conditions are fullfilled, then any cycle (= smooth closed curve) or path joining two fixed endpoints in $R^{N}-S$ can be continuously deformed into a solution of (1), provided that the cycle or path winds around $S$ in a sufficiently complicated way. Specifically, we have the following definitions:

Definition 1. A cycle $\gamma$ in $R^{N}-S$ will be said to be tied to $S$ if $\gamma$ cannot be continuously moved off to infinity without either crossing $S$ or having its arc length become infinite. (See below for examples.)

Definition 2. A path $\gamma$ joining two fixed points $p, q$ in $R^{N}-S$ will be said to be (topologically) simple if $p=q$ and $\gamma$ is homotopic in $R^{N}-S$ to a point, or if $p \neq q$ and $\gamma$ belongs to the homotopy class of paths in $R^{N}-S$ which contains the line segment $\overline{p q}$. (This homotopy class will be understood to be empty if $\overline{p q}$ intersects $S$.)

We can now state our principal existence theorems.

[1a] Assuming [A] and [B], and given any $\varepsilon>0$, then any cycle $\gamma$ which is tied to $S$ can be continuously deformed into a periodic solution of (1) which at some points comes to within an $\varepsilon$-distance of $S$; moreover, $\gamma$ does not cross $S$ during the deformation. I.e., $\gamma$ is homotopic in $R^{N}-S$ to an infinite number of periodic solutions to (1) which cut arbitrarily small neighborhoods of $S$.

[1b] Assuming [A] and [C], and given any $\lambda>0$, then any cycle $\gamma$ which is tied to $S$ is homotopic in $R^{N}-S$ to a $\lambda$-periodic solution of (1).

[2a] Assuming [A] and [B], then every smooth nonsimple path $\gamma$ joining two fixed points $p, q$ is homotopic in $R^{N}-S$ to an infinite number of solutions of (1) which join $p$ to $q$ and cut arbitrarily small neighborhoods of $S$.

[2b] Assuming [A] and [C], and given any $t_{1}>0$, then every smooth nonsimple path $\gamma$ joining $p$ to $q$ is homotopic in $R^{N}-S$ to a solution of (1) which joins $p$ to $q$ in time $t_{1}$.

ExAMPLES. (i) Let $N=2$ and let $S$ be a discrete set of points. In this particular case a cycle is tied to $S$ if and only if it is not homotopic (in $R^{2}-S$ ) to a point; but this is not a general fact as the following example will show.

(ii) Let $N=3$ and let $S$ be the union of two intersecting lines. A cycle is tied to $S$ if and only if it winds around both of the lines. A cycle which winds around only one of the lines is not tied to $S$.

(iii) Let there be three masses $m_{i}$ with position coordinates $\left(x_{i}, y_{i}\right)$ con- 
strained to move in the plane $R^{2}$, so that the configuration space of the system is $R^{6}$. We suppose that each mass attracts every mass along the lines joining them. Reduce the dimension of the configuration space from six to four by fixing the centroid at the origin. The singularities of the system correspond to collisions of the masses, and these are defined by the three coincidence relations $P_{i}:\left\{x_{j}=x_{k}\right.$ and $\left.y_{j}=y_{k}\right\}$, where the triple $\{i, j, k\}$ varies over the cyclic permutations of $\{1,2,3\}$. Hence the configuration space of the reduced system with the singularities removed is $R^{4}-S$ where $S$ is the union of the three 2-planes $P_{i}$. Any pair of these planes intersect at precisely one point (the origin). A cycle $\gamma$ is tied to $S$ if and only if it winds around at least two of the planes. The property of winding around the plane $P_{i}$ can be defined by the nonvanishing of the period $\int_{\gamma} \omega_{i}$ where $\omega_{i}$ is the closed but nonexact differential 1-form which corresponds to an infinitesimal rotation around the plane $P_{i}$. (Similar remarks apply to planar $n$-body systems, $n>3$.)

3. Sketch of proofs. Our discussion will be confined to the periodic cases [1a], [1b]. For any cycle $\gamma$ given in euclidean coordinates by $x=x(t)$, let $J(\gamma)=\frac{1}{2} \int|\dot{x}(t)|^{2} d t, E(\gamma)=\int V(x(t)) d t$ and $L(\gamma)=J(\gamma)-E(\gamma)$, where the integrations are taken over a period. Fix a number $\lambda>0$, and let $H^{1}$ denote the Sobolev space of all absolutely continuous $\lambda$-periodic cycles $\gamma$ in $R^{N}$ for which $J(\gamma)<\infty$. An admissible norm for $H^{1}$ is given by $\|\cdot\|_{1}$ where $\|\gamma\|_{1}^{2}=\int|x(t)|^{2} d t+\int|\dot{x}(t)|^{2} d t$. (See [5] for details and references.) The space of all $\lambda$-periodic cycles $\gamma$ in $R^{N}-S$ of class $H^{1}$ will be denoted by $M$, and for $c>0$ we set $M^{c}=M \cap J^{-1}(c)$.

Let $X$ be a smooth Riemannian manifold which is possibly infinite dimensional. Recall that a $C^{1}$ function $f$ on $X$ which is bounded above (below) is said to satisfy the P-S condition (often referred to as "Condition $C$ ") if every subset of $X$ on which $f$ is bounded and $|\nabla f|$ is not bounded away from zero contains a critical point of $f$ in its closure. It is known that the Morse and Lusternik-Schnirelman critical point theories can be applied to functions satisfying this condition provided that $X$ is complete; in particular, such functions attain absolute maximum (minimum) values on each component of $X$ ([7], [8]). If $X$ is not complete, the following condition is required for the theory to hold [8, p. 208].

[D] For every real number $a, f^{-1}[a, \infty)\left(f^{-1}(-\infty, a]\right)$ is complete.

The proofs of statements [1a] and [1b] consist in showing that the functionals $E \mid M^{c}$ and $L \mid M$ satisfy the P-S condition, and the solutions obtained are cycles at which these functionals attain maximum and minimum values resp. (Thus, in the case [1b] we prove a strong form of Hamilton's Principle.) The manifolds $M^{c}$ and $M$ are not complete because 
of the existence of "holes" in the underlying configuration space $R^{N}-S$, and the crucial part of the proofs is to render this lack of completeness ignorable; i.e. we show assumption [A] implies property [D]. (For an account of how this is done see [6].)

The device of exhibiting solutions of (1) as extremals for $E \mid M^{c}$ is due to M. S. Berger [1], [2], [3] and has been used by him and the author [5] to obtain information concerning the existence of periodic solutions to conservative systems with convex potentials. When written out in coordinates the relation $\delta\left(E \mid M^{c}\right)=0$ becomes $\mu \ddot{x}(t)+\nabla V(x(t))=0$, where $\mu$ is a Lagrange multiplier. If $\mu>0$, this relation reduces to (1) after the change of scale $t \rightarrow t \sqrt{\mu}$, and it turns out that [B] insures that $\mu>0$. In particular cases we can often dispense with this assumption (cf. [6]).

Let $\gamma$ be a $\lambda$-periodic cycle which winds around $S$. From an elementary application of the Schwarz inequality we get the relation arc length $(\gamma)$ $\leqq(2 \lambda J(\gamma))^{1 / 2}$. Therefore decreasing $J(\gamma)$ has the effect of tightening the loops of $\gamma$ around $S$. This is how we obtain solutions which cut arbitrarily small neighborhoods of $S$.

4. The gravitational case. (i) For simplicity let $N=2$ and $V(x)$ $=-|x|^{1 / 2}$. To see why $[\mathrm{A}]$ excludes the gravitational case, suppose that $U$ satisfies (2). Then $U$ can behave no worse than $|x|^{1 / 2}$, which contradicts the requirement that $U$ have an infinitely deep well at the origin.

(ii) To show that the P-S condition is not satisfied by, say, $L$ defined on $M$, we consider a sequence of equi-energy elliptical orbits $\left\{\gamma_{n}\right\}$ whose perihelions converge to the origin. It is known that equi-energy orbits have equal periods, and also equal averaged kinetic energies $J(\gamma)$ and equal averaged potential energies $E(\gamma)$. (E.g. see [4].) Hence $L\left(\gamma_{n}\right)=$ const (and $\delta L\left(\gamma_{n}\right)=0$ ). Hence $L$ does not satisfy property [D]. (Also, by perturbing each $\gamma_{n}$ in a neighborhood of its aperihelion, we obtain a sequence $\left\{\gamma_{n}^{\prime}\right\}$ for which $\left|L\left(\gamma_{n}^{\prime}\right)\right|$ is bounded, $\nabla L\left(\gamma_{n}^{\prime}\right) \rightarrow 0$, and such that $\left\{\gamma_{n}^{\prime}\right\}$ contains no convergent subsequence.)

(iii) Let $p$ and $q$ be two fixed points distinct from the origin. At the end of $\$ 3$ we showed how our theorems provide for the existence of trajectories which join $p$ to $q$ and make arbitrarily tight loops around the singularity at the origin. Such solutions do not exist in the gravitational case.

5. Finally, we mention another method of proving the existence theorems of $\S 2$. Recall that a function $f$ defined on a Hilbert space $H$ is lower semicontinuous in the weak topology of $H$ if $f^{-1}(-\infty, c]$ is weak closed for every $c$, and that such a function is bounded below and attains its minimum on every weakly compact subset of $H$. Hence if $f^{-1}(-\infty, c]$ is weakly compact for every $\bar{c}$, it follows that $f$ is bounded below (on $H$ ) and attains 
its minimum. Moreover, for such a function $f$, if $M$ is a weak closed subset of $H$, then $f \mid M$ attains its minimum on $M$. Using this principle one can prove the existence theorems of $\$ 2$, assumption [A] this time being necessary to establish the weak closure of the various manifolds $M$ which occur in the arguments.

\section{REFERENCES}

1. Melvyn S. Berger, On periodic solutions of second order Hamiltonian systems. I, J. Math. Anal. Appl. 29 (1970), 512-522. MR 41 \# 2121.

2. - Multiple solutions of nonlinear operator equations arising from the calculus of variations, Proc. Sympos. Pure Math., vol. 18, part 1, Amer. Math. Soc., Providence, R:I., 1970, pp. 10-27. MR 42 \#5112.

3. tional problems, Amer. J. Math. 93 (1971), 1-10. MR 43 \# 2588.

4. W. B. Gordon, On the relation between period and energy in periodic dynamical systems, J. Math. Mech. 19 (1970), 111-114. MR 39 \# 7236.

5. - A theorem on the existence of periodic solutions to Hamiltonian systems with convex potential, J. Differential Equations 10 (1971), 324-335.

6. - Periodic solutions to Hamiltonian systems with infinitely deep potential wells, Ordinary Differential Equations, Academic Press, New York, 1972, pp. 399-403.

7. R. S. Palais, Morse theory on Hilbert manifolds, Topology 2 (1963), 299-340. MR $28 \# 1633$

8. , Critical point theory and the minimax principle, Proc. Sympos. Pure Math., vol. 15, Amer. Math. Soc., Providence, R.I., 1970, pp. 185-212. MR 41 \# 9303.

Mathematics Research Center, Naval Research laboratory, Washington, D.C. 20390 